\title{
Contribución al estudio de la resistencia química de los cementos. Comportamiento mecánico de un cemento portland resistente al yeso (1. ${ }^{\mathrm{a}}$ Parte)
}

\author{
DEMETRIO GASPAR-TEBAR, JOSE LUIS SAGRERA-MORENO y \\ Drs. en Ciencias Químicas (IETCC/CSIC) \\ VICTORIANO GONZALES-VILA \\ (Cementos del Atlántico, S. A.)
}

\section{$R E S U M E N$}

En el presente trabajo, primera parte de otro en el que se dará cuenta de la evolución de las resistencias mecánicas de diversas series de probetas de mortero $y$ de hormigón sometidas a la acción de distintas disoluciones agresivas, se estudia el comportamiento quimico-resistente de un cemento portland industrial resistente al yeso (con un contenido calculado, Bogue, nulo de $C_{3} A$, del $10,8 \%$ de $C_{4} A F$ y del $81,7 \%$ de $\left.\mathrm{C}_{3} \mathrm{~S}+\mathrm{C}_{2} \mathrm{~S}\right)$ frente al $\mathrm{CaSO}_{4} .2 \mathrm{H}_{2} \mathrm{O}$ por los métodos de Le Chatelier-Anstett y el descrito en la norma ASTM C 452-73 y frente a las siguientes disoluciones agresivas: disolución saturada de sulfato de calcio, de $\mathrm{Na}_{2} \mathrm{SO}_{4}(2,1 \mathrm{~g} / \mathrm{l})$, de $\mathrm{MgSO}_{4} .7 \mathrm{H}_{2} \mathrm{O}(3,22 \mathrm{~g} / \mathrm{l})$, de $\mathrm{MgSO}_{4} .7 \mathrm{H}_{2} \mathrm{O}(32,2 \mathrm{~g} / \mathrm{l})$, saturada de sulfato de calcio $+\mathrm{MgSO}_{4} .7 \mathrm{H}_{2} \mathrm{O}(3,22 \mathrm{~g} / \mathrm{l})$, saturada de sulfato de calcio $+\mathrm{MgSO}_{4} \cdot 7 \mathrm{H}_{2} \mathrm{O}(32,2 \mathrm{~g} / \mathrm{l})$, de $\mathrm{Na}_{2} \mathrm{SO}_{4}(3,5 \mathrm{~g} / \mathrm{l})+\mathrm{NaCl}(20,0 \mathrm{~g} / \mathrm{l})$, agua de mar artificial ASTM D 1141-75 y, además, agua potable filtrada por los métodos de Wittekindt y de Koch-Steinegger, durante diversos periodos de tiempo, lo que ha permitido establecer una clasificación del cemento estudiado frente a las mencionadas disoluciones agresivas, según su comportamiento.

\section{$S U M M A R Y$}

This study, first part of a work where an account of the evolution of the mechanical resistances of various series of mortar and concrete tests submitted to the action of different agressive solutions will be given, sets up the chemical resistant behaviour of a sulphate resistant portland cement (with a content calculated -Bogue- null of $C_{3} A$, of $10,8 \%$ of $C_{4} A F$ and of $81,7 \%$ of $\mathrm{C}_{3} \mathrm{~S}+\mathrm{C}_{2} \mathrm{~S}$ ) against the $\mathrm{CaSO}_{4} \cdot 2 \mathrm{H}_{2} \mathrm{O}$ through the Le Chatelier-Anstett methods, and described in the ASTM C 452-73 and against the following agressive solutions: saturated solution calcium sulphate, $\mathrm{Na}_{2} \mathrm{SO}_{4}(2,1 \mathrm{~g} / \mathrm{l}), \mathrm{MgSO}_{4} .7 \mathrm{H}_{2} \mathrm{O}(3,22 \mathrm{~g} / \mathrm{l})$, $\mathrm{MgSO}_{4} .7 \mathrm{H}_{2} \mathrm{O}(32,2 \mathrm{~g} / \mathrm{l})$, saturated of calcium sulphate $+\mathrm{MgSO}_{4} .7 \mathrm{H}_{2} \mathrm{O}(3,22 \mathrm{~g} / \mathrm{l})$, saturated of calciun sulphate $+\mathrm{MgSO}_{4} .7 \mathrm{H}_{2} \mathrm{O}(32,2 \mathrm{~g} / \mathrm{l})$,

$\mathrm{Na}_{2} \mathrm{SO}_{4}(3,5 \mathrm{~g} / \mathrm{l})+\mathrm{NaCl}(20,0 \mathrm{~g} / \mathrm{l})$, artificial sea water and, also, filtered drinking water, through of the Wittekindt and Kock Steinegger methods, during diverse periods, which allowed to establish a classification, according to his behaviour, of the studied cement against those agressive solutions.

\section{INTRODUCCION}

En otros trabajos (1) (2) (3) (4) (5) (6), se ha estudiado:

a) La evolución de las resistencias mecánicas a flexotracción de las probetas de mortero $(1: 3)$ de $1 \times 1 \times 6 \mathrm{~cm}$, hechas con el cemento portland industrial resistente al yeso (cemento $2<>$ P-450-Y), objeto del presente artículo, sumergidas en agua potable filtrada $\left(^{*}\right)(1)$, unás series de 12 probetas, en agua de mar artificial ASTM 1141-75, otras series análogas $(* *)(1)$, así como en una disolución saturada de yeso $(* * *)(2)$, durante 56 - 90 - 180 y 360 dias, después de haberlas curado 24 horas en un recinto saturado de humedad y, a continuación, bajo agua potable filtrada hasta 22 dias.

(*) Sistema: cemento 2-agua potable filtrada.

(**) Sistema: cemento 2-agua de mar artificial.

(***) Sistema: cemento 2-disolución saturada de yeso. 
b) La composición estructural de las nuevas fases sólidas formadas en los medios de curado y de conservación (agua potable filtrada) (3), así como de conservación-ataque (agua de mar artificial) (4), en donde han estado sumergidas las diversas series de probetas de mortero hasta 360 días.

c) La evolución de las características estructurales de la fracción enriquecida (cemento hidratado y cemento hidratado-atacado), extraida de uno de los prismas de mortero de cada serie de probetas de los sistemas cemento 2 -agua potable filtrada (3) y cemento 2 -agua de mar artificial (4).

d) La variación de la concentración de los iones calcio y sulfato en los medios de curado y de conservación (agua potable filtrada) (5) y de conservación-ataque (6), en donde han estado sumergidas las diversas series de probetas de mortero durante los períodos de tiempo mencionados anteriormente, así como de las fases sólidas formadas, y la evolución del $\mathrm{pH}$ de dichos medios. Además de la concentración de los iones señalados, en el sistema cemento 2-agua de mar artificial, se ha determinado el contenido de los iones $\mathrm{Mg}$ (II) y $\mathrm{Cl}(\mathrm{I})$.

En el artículo presente, se estudia el comportamiento quimico resistente de un cemento portland industrial (P-450-Y) de fabricación española, análogo al utilizado en los trabajos reseñados, frente al sulfato de calcio dihidratado por los métodos de Le Chatelier-Anstett, por el descrito en la norma ASTM C 452-73 y frente a las disoluciones agresivas, que se incluyen en el apartado 2, por los métodos de Wittekindt y de Koch-Steinegger; todos ellos hasta la edad de 1 año. A la vista de los resultados obtenidos se amplió el estudio correspondiente al método de KochSteinegger, utilizando las disoluciones agresivas que se señalan en dicho apartado, hasta la edad de 3 años.

\section{PARTE EXPERIMENTAL}

En este trabajo se estudia el comportamiento quimico-resistente de un cemento portland industrial resistente al yeso, de fabricación española, cuando se somete a la acción de las siguientes disoluciones agresivas:

Disolución 1. Disolución saturada de sulfato de calcio $\left(\simeq 2,1 \mathrm{~g} / l\right.$ de $\left.\mathrm{CaSO}_{4} \cdot 2 \mathrm{H}_{2} \mathrm{O}\right)$.

Disolución 2. Disolución de $\mathrm{Na}_{2} \mathrm{SO}_{4}(2,1 \mathrm{~g} / l)$.

Disolución 3. Disolución de $\mathrm{MgSO}_{4} \cdot 7 \mathrm{H}_{2} \mathrm{O}(3,22 \mathrm{~g} / l)$.

Disolución 4. Disolución de $\mathrm{MgSO}_{4} \cdot 7 \mathrm{H}_{2} \mathrm{O}(32,2 \mathrm{~g} / l)$.

Disolución 5. Disolución saturada de sulfato de calcio $+\mathrm{MgSO}_{4} \cdot 7 \mathrm{H}_{2} \mathrm{O}(3,22 \mathrm{~g} / l)$.

Disolución 6. Disolución saturada de sulfato de calcio $+\mathrm{MgSO}_{4} \cdot 7 \mathrm{H}_{2} \mathrm{O}(32,2 \mathrm{~g} / l)$.

Disolución 7. Disolución de $\mathrm{Na}_{2} \mathrm{SO}_{4}(3,5 \mathrm{~g} / l)+\mathrm{NaCl}(20,0 \mathrm{~g} / l)$.

Disolución 8. Agua de mar artificial ASTM D 1141-75 y, además,

Disolución 0. Agua potable filtrada.

determinando:

a) Las caracteristicas quimicas, estructurales, físicas y mecánicas del cemento.

b) La resistencia química de dicho cemento, en forma de pasta y en forma de mortero, por el método de Le Chatelier-Anstett y por el descrito en la norma ASTM C 452-73. Los periodos de tiempo estudiados han sido: $1,7,14,28,90,180$ y 360 días en el primer método y $7,14,21,30,60,90,150,180,210,270,300$ y 360 días en el segundo. 
c) La resistencia química del cemento mencionado, en forma de mortero, por los métodos de Wittekindt y de Koch-Steinegger frente a las disoluciones 1, 2, 3, 7 y 8, en donde se han sumergido las probetas de mortero después del periodo de curado (24 horas en cámara húmeda y hasta 22 dias bajo agua potable filtrada), durante $28,84,112,140,168,196$, $280,308,336$ y 364 días en el primer método y durante 56, 180 y 360 días en el segundo.

Las series de probetas de mortero testigo se han sumergido durante los mismos períodos de tiempo en agua potable filtrada, que se ha designado disolución 0 .

d) La resistencia química de probetas de mortero $(1: 3)$ de $1 \times 1 \times 6 \mathrm{~cm}$ (método de KochSteinnegger), hechas con el cemento citado frente, a las disoluciones agresivas $1,3,4,5,6$, $8 \mathrm{y}$, además, agua potable filtrada, que se ha designado disolución 0 ; para ello se han sumergido diversas series de probetas de mortero (12 probetas por edad, por agresivo y por serie) en las distintas disoluciones agresivas, después del periodo de curado $(1+21$ días), durante $56-90-180-360-540-720-900$ y 1.080 días.

\subsection{Materiales utilizados}

\section{a) Cemento}

Se ha utilizado un cemento portland industrial resistente al yeso, de fabricación española, que responde a las características químicas, fisicas y mecánicas de la tabla 1 .

En la figura 1 se encuentran los DRX del cemento anhidro y del residuo procedente del ataque de dicho cemento con ácido salićlico-metanol (7), para $2 \theta$ comprendidos entre 30 y $35^{\circ}$, el primero, y $32-35^{\circ}$, el segundo.

\section{T ABLA 1}

Composición química; composición potencial calculada (Bogue) y características fisicas y mecánicas del cemento

\section{Composición quimica}

$$
\text { Determinaciones }
$$

\section{\% en masa}

Perdida por calcinación, P.F. . . . . $\ldots \ldots \quad 1,1$

Residuo insoluble, R.I. ........... 0,2

Dióxido de silicio, $\mathrm{SiO}_{2} \ldots \ldots \ldots \ldots \ldots \ldots$

Oxido de hierro (III), $\mathrm{Fe}_{2} \mathrm{O}_{3} \ldots \ldots \ldots \ldots \quad 4,1$

Oxido de aluminio, $\mathrm{Al}_{2} \mathrm{O}_{3} \ldots \ldots \ldots \ldots \ldots \quad 1,8$

Oxido de calcio, $\mathrm{CaO} \ldots \ldots \ldots \ldots \ldots .67,9$

Oxido de magnesio, $\mathrm{MgO} \ldots \ldots \ldots \ldots \ldots, 0,5$

Trióxido de azufre, $\mathrm{SO}_{3} \ldots \ldots \ldots \ldots \ldots \ldots, 2,3$

Suma $\ldots \ldots \ldots \ldots \ldots \ldots$.

Oxido de calcio libre, $\mathrm{CaO}$ libre $\ldots \ldots \ldots \ldots \quad 1,8$

\section{Composición potencial calculada (Bogue)}

Silicato tricálcico, $\mathrm{C}_{3} \mathrm{~S} \quad \ldots \ldots \ldots \ldots \ldots \ldots \quad 75,6$

Silicato bicálcico, $\mathrm{C}_{2} \mathrm{~S} \ldots \ldots \ldots \ldots \ldots \ldots \ldots, 6,1$

Aluminato tricálcico, $\mathrm{C}_{3} \mathrm{~A} \ldots \ldots \ldots \ldots \ldots \quad 0,0$

Ferrito aluminato tetracálcico, $\mathrm{C}_{4} \mathrm{AF} \ldots \ldots \quad 10,8$

Sulfato de calcio, $\mathrm{CaSO}_{4} \ldots \ldots \ldots \ldots \ldots \quad 3,9$

\section{Caracteristicas fisicas y mecánicas}

Superficie especifica Blaine, $\mathrm{cm}^{2} / \mathrm{g} \ldots 3.553$

Peso especifico, $\mathrm{g} / \mathrm{cm}^{3} \ldots \ldots \ldots \ldots \ldots \quad 3,12$

Expansión autoclave, $\% \ldots \ldots \ldots \ldots \quad 0,02$

Agua de consistencia, \% ........... $\quad 25,6$

Tiempo de fraguado:

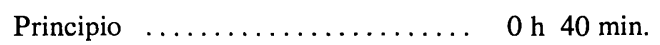

Final ................... 3 h 15 min.

Resistencias mecánicas, $\mathrm{kp} / \mathrm{cm}^{2}$ :

Flexotracción:

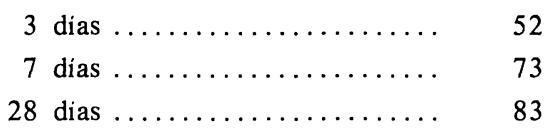

Compresión:

3 dias ................... 285

7 dias .................... 473

28 días .................... 542 
b) Arena utilizada para preparar las probetas de mortero de $1 \times 1 \times 111 / 4$ " (ensayo ASTM C 452-73)

Se ha utilizado la arena especificada en la norma ASTM C 452-73 (arena normalizada de Ottawa).

c) Arena empleada para fabricar las probetas de mortero de $4 \times 4 \times 1 \mathrm{~cm}$ (ensayo de Wittekindt)

Se ha empleado la arena especificada en el Pliego de Prescripciones Técnicas Generales para la Recepción de Cementos (RC-75), en la preparación de morteros normalizados (RC-75).
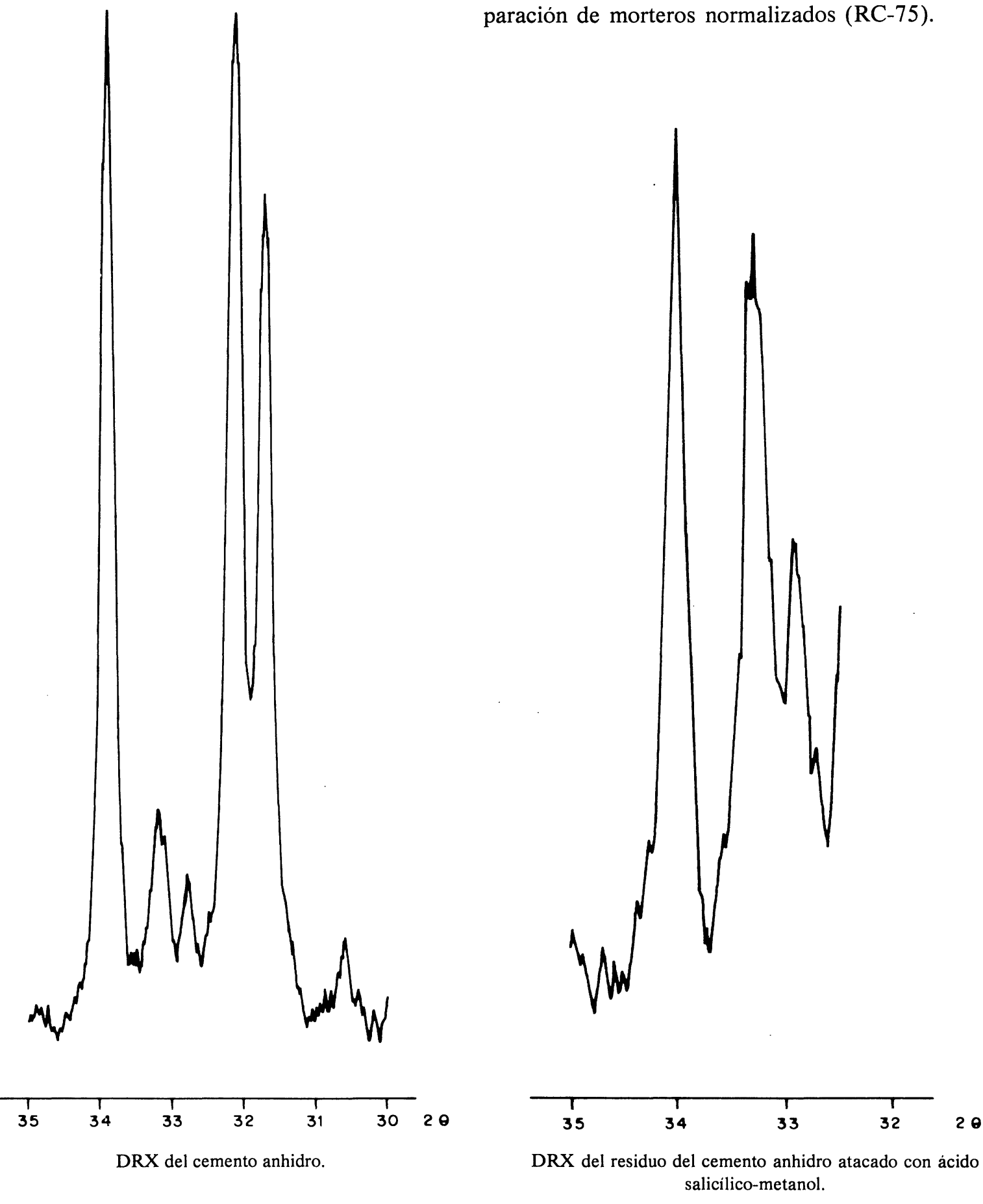

Fig. 1.-DRX del cemento anhidro y del residuo de dicho cemento anhidro atacado con ácido salicilico-metanol. 
d) Arena usada para preparar las probetas de $1 \times 1 \times 6 \mathrm{~cm}$ (ensayo de Koch-Steinegger)

Se ha usado la arena natural (El Espirdo-Segovia) análoga a la empleada para preparar los morteros normalizados (RC-75), con un contenido de $\mathrm{SiO}_{2}$ superior al $99 \%$. El tamaño de grano está comprendido entre 1 y $1,5 \mathrm{~mm}$.

\subsection{Preparación de las disoluciones agresivas}

Disolución 1. Disolución saturada de sulfato de calcio, con una concentración de $\mathrm{SO}_{4}(\mathrm{II})=$ $=1,100 \mathrm{~g} / 1$

La disolución saturada de sulfato de calcio se ha preparado disolviendo yeso natural (tiene una riqueza de $\mathrm{CaSO}_{4} .2 \mathrm{H}_{2} \mathrm{O}$ superior a $98 \%$, determinada por vía química y por DRX) en agua potable filtrada hasta saturación. Posteriormente, se ha determinado la concentración de iones sulfato y la de iones calcio; los resultados obtenidos se incluyen en la tabla 2.

T AB LA 2

Concentración (g/l) de los iones $\mathrm{SO}_{4}(\mathrm{II}), \mathrm{Cl}(\mathrm{I}), \mathrm{Ca}$ (II) y $\mathrm{Mg}$ (II) de las disoluciones agresivas y del agua potable filtrada

\begin{tabular}{|l|c|c|c|c|}
\hline \multirow{2}{*}{ Disolución n. } & \multicolumn{4}{|c|}{ Concentración, $\mathbf{g} / \mathbf{1}$} \\
\cline { 2 - 5 } & $\mathbf{S O}_{\mathbf{4}}$ (II) & $\mathbf{C l}$ (I) & $\mathbf{C a}$ (II) & $\mathbf{M g}$ (II) \\
\hline & & & & \\
2 & 1,010 & - & 0,420 & - \\
3 & 1,210 & - & - & - \\
4 & 1,263 & - & - & 0,318 \\
5 & 12,630 & - & - & 3,180 \\
6 & 2,273 & - & 0,420 & 0,318 \\
7 & 13,280 & - & 0,420 & 3,180 \\
8 & 2,494 & 12,132 & - & - \\
0 (agua potable filtrada) & 2,928 & 19,585 & 0,521 & 1,347 \\
& 0,003 & 0,007 & 0,005 & 0,0016 \\
\hline
\end{tabular}

\section{Disoluciones 2, 3, 4}

Estas disoluciones se han preparado, a partir de productos p. a. (Probus), disolviendo en agua potable filtrada $2,1 \mathrm{~g} / l$ de $\mathrm{Na}_{2} \mathrm{SO}_{4} ; 3,22 \mathrm{~g} / l$ de $\mathrm{MgSO}_{4} .7 \mathrm{H}_{2} \mathrm{O}$ y $32,2 \mathrm{~g} / l$ de $\mathrm{MgSO}_{4} .7 \mathrm{H}_{2} \mathrm{O}$, respectivamente. A continuación, se ha determinado en dichas disoluciones la concentración de iones sulfato y la de iones magnesio; los valores obtenidos figuran en la tabla 2 .

\section{Disoluciones 5 y 6}

Las disoluciones 5 y 6 se han preparado a partir de la disolución saturada de sulfato de calcio, en la que se han disuelto $3,22 \mathrm{~g} / l$, para la primera disolución, y $32,2 \mathrm{~g} / l$, para la segunda disolución, de $\mathrm{MgSO}_{4} .7 \mathrm{H}_{2} \mathrm{O}$ (Probus, p.a.). Una vez preparadas dichas disoluciones se ha determinado la cocentración de los iones sulfato, calcio y magnesio, cuyas concentraciones se incluyen en la tabla 2. 


\section{Disolución 7}

Esta disolución se ha preparado disolviendo en agua potable filtrada $3,5 \mathrm{~g} / l$ de $\mathrm{Na}_{2} \mathrm{SO}_{4}$ (Probus, p.a.) y $20,0 \mathrm{~g} / l$ de $\mathrm{NaCl}$ (Probus, p.a.). Como en las disoluciones anteriores se ha determinado, posteriormente, las concentraciones de los iones sulfato y cloruro; los valores obtenidos se encuentran en la tabla 2 .

\section{Disolución 8. Agua de mar artificial}

El agua de mar artificial se ha preparado según el punto 6 de la norma ASTM D 1141-75 (8) que se recoge en (9), utilizando las disoluciones 1 y 2 . En este agua de mar artificial, que se ha designado ASTM D 1141-75, se ha determinado el contenido de los iones calcio, magnesio, sulfatos y cloruros, así como el valor del $\mathrm{pH}$; dichos contenidos son los que se indican en la tabla 2.

Para preparar las mencionadas disoluciones 1 y 2 se han utilizado productos Probus, p.a. y agua potable filtrada.

\section{Disolución 0. Agua potable filtrada}

Como medio de curado para todas las series de probetas de mortero y de conservación para las series testigo, se ha utilizado agua potable (canal Isabel II - Madrid), una vez que ha pasado a través de un filtro cerámico. El contenido de los iones calcio, magnesio, sulfatos y cloruros es el que figura en la tabla 2.

\subsection{Procedimientos operatorios}

Los procedimientos operatorios utilizados han sido los siguientes:

a) Las características químicas, físicas y mecánicas del cemento por los procedimientos que figuran en el Pliego (RC-75) (10),

b) el residuo insoluble del cemento en ácido salicilico-metanol por la técnica recogida en (7),

c) los diagramas de difracción de rayos $\mathrm{X}$ del cemento y del residuo en ácido salicílico-metanol, se han obtenido por medio de un equipo Philips PW-1010, con una unidad de registro PW-1050, trabajando en las condiciones reseñadas en (11),

d) el contenido de los iones $\mathrm{Ca}$ (II) y $\mathrm{Mg}$ (II) por complexometría con EDTA, el de los iones $\mathrm{SO}_{4}$ (II) por gravimetría al estado de $\mathrm{BaSO}_{4}$ y el de los iones $\mathrm{Cl}$ (I) volumétricamente por el método de Mohr,

e) la resistencia quimica del mencionado cemento resistente al yeso, se ha obtenido por los procedimientos operatorios descritos en (12) para el método Le Chatelier-Anstett, en (13) para el método de la norma ASTM C 452-73, en (15) para el método de Wittekindt y en (14) para el método de Koch-Steinegger, métodos que se recopilan y estudian en (15).

\section{RESULTADOS Y DISCUSION}

\subsection{Caracteristicas del cemento}

El cemento estudiado en este trabajo, tiene un contenido calculado (Bogue) de $\mathrm{C}_{3} \mathrm{~A}=0 \%$ y de 
$\mathrm{C}_{4} \mathrm{AF}=10,8$; el valor correspondiente a la suma $\mathrm{C}_{3} \mathrm{~S}(75,6 \%)$ y $\mathrm{C}_{2} \mathrm{~S}(6,1 \%)$ es $81,7 \%$. Las fases cristalinas correspondientes a los aluminatos del cemento anhidro y del residuo del ataque del cemento anhidro con ácido salicilico-metanol se pueden apreciar en los DRX de la figura 1 , en los que se ha detectado un pico del $\mathrm{C}_{3} \mathrm{~A}$ a $2 \theta=33,15^{\circ}$ y otro del $\mathrm{C}_{4} \mathrm{AF}$ a $2 \theta=33,8^{\circ}$ ( $34^{\circ}$ teóricos); además se ha puesto de manifiesto la existencia de otro pico a $2 \theta=32,9^{\circ}$ perfectamente diferenciado en el DRX del residuo del cemento tratado con ácido salicílicometanol. Este cemento, por sus características químicas y mecánicas, se puede clasificar de acuerdo con el Pliego RC-75 como P-450-Y.

\subsection{Resistencia química}

\subsubsection{Método de Le Chatelier-Anstett}

Los valores obtenidos, variación experimentada por el diámetro de las probetas (dos) - cemento hidratado más yeso dihidratado-, expresados en tanto por ciento, se han representado en función del tiempo en la figura 2. En dicha figura se observa que el valor alcanzado a los 14 días $(0,12 \%)$ permanece constante a lo largo de las edades estudiadas $(7,14,28,90,180$ y 360 días).

En las mencionadas probetas no se han observado grietas, ni deformaciones; la penetración de la sonda ha sido nula.

El incremento de diámetro máximo especificado por este método, para considerar que un cemento es resistente a la acción del sulfato de calcio, es $1,25 \%$ a los 28 días.'

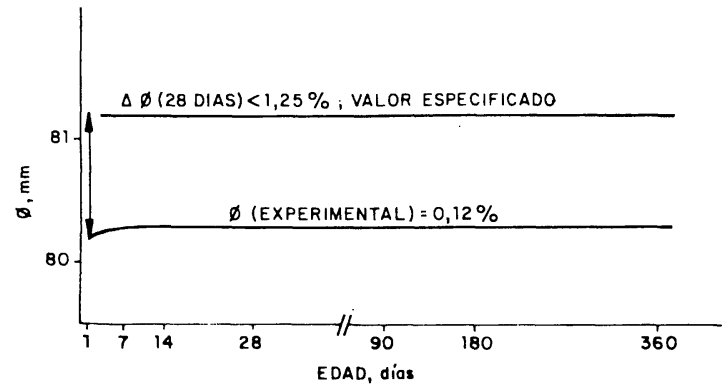

Fig. 2.-Evolución del incremento de diámetro (método Le Chatelier-Anstett).

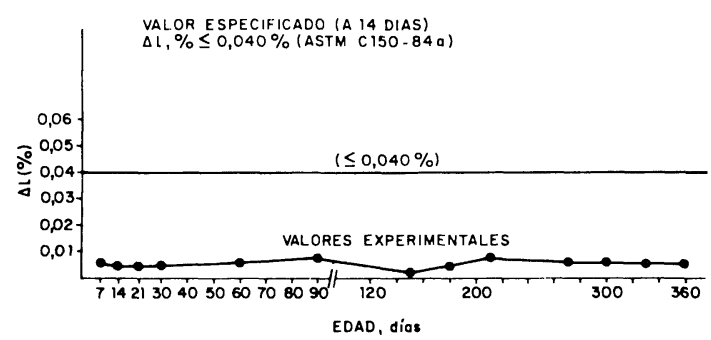

Fig. 3.-Evolución del incremento de longitud (método ASTM C 452-73).

\subsubsection{Método de ensayo descrito en la norma ASTM C 452-73}

El incremento de longitud $(\triangle l)$ experimentado por las dos series de probetas de mortero, con relación al de las probetas originales, expresado en $\%$, a los 14 días ha sido $0,004 \%$. A todas las edades estudiadas dicho incremento ha sido menor de 0,009 \%, como se puede apreciar en la figura 3 en donde se ha representado el $\Delta l$, en $\%$, en función del tiempo $(7,14,21,30,60$, $90,150,180,210,270,300,330$ y 360 días).

El incremento de longitud, determinado por el procedimiento descrito en la mencionada norma ASTM C 452-73, especificado para los cementos tipo V (cementos de alta resistencia a la acción de los sulfatos), según la norma ASTM C 150-84, debe ser igual o menor de 0,045 \% a los 14 días y de $0,040 \%$, según la norma C $150-84 a$, a la misma edad; valores muy superiores a los obtenidos con el cemento estudiado. 


\subsubsection{Método de Wittekindt}

Las variaciones de longitud que han experimentado las diversas series de probetas de mortero (6 por serie) sumergidas en las diferentes disoluciones agresivas, excepto para el agua de mar artificial ASTM D 1141-75, señaladas en el punto c) del apartado 2, son prácticamente constantes y del mismo orden que las correspondientes a las series análogas de probetas sumergidas en agua potable filtrada, para todas las edades estudiadas $(28,84,112,140,168,196,280,308$, 336 y 364 días).

En el caso de las probetas sumergidas en agua de mar artificial ASTM D 1141-75, éstas han experimentado, a todas las edades, una retracción comprendida entre 0,01 y $0,03 \mathrm{~mm}$, que corresponde a 0,06 y $0,19 \mathrm{~mm} / \mathrm{m}$, respectivamente, y a 0,006 y $0,019 \%$, con relación a la longitud inicial de las probetas.

\subsubsection{Método de Koch-Steinegger}

Los valores de los coeficientes de corrosión Koch-Steinegger $\left(R_{t} / R^{\prime}\right)$ de las distintas series de probetas de mortero $(1: 3)$ de $1 \times 1 \times 6 \mathrm{~cm}(12$ probetas por edad y por disolución), sumergidas en las disoluciones agresivas que se relacionan en el punto c) del apartado 2 , durante 56 - 180 y 360 días después del período de curado $(1+21$ dias $)$, se han representado, en función del tiempo de conservación, en la figura 4.

Dichos coeficientes de corrosión para la edad $t=22+56$ días son superiores al valor indice $(0,70)$ especificado para calificar a un cemento como resistente - desde un punto de vista de resistencia química - frente a un medio agresivo dado.

En la figura 4 se aprecia la evolución de los mencionados coeficientes de corrosión a lo largo del tiempo, lo que ha permitido establecer la siguiente escala (de mayor a menor) según su comportamiento frente a las cinco disoluciones agresivas estudiadas:

Disolución saturada de yeso $>$ Disolución de $\mathrm{MgSO}_{4} \cdot 7 \mathrm{H}_{2} \mathrm{O}(3,22 \mathrm{~g} / l)>$ Disolución de $\mathrm{Na}_{2} \mathrm{SO}_{4}$ $(2,1 \mathrm{~g} / l)>$ Disolución de $\mathrm{Na}_{2} \mathrm{SO}_{4}(2,1 \mathrm{~g} / l)$ más $\mathrm{NaCl}(20,0 \mathrm{~g} / l)>$ Agua de mar artificial ASTM D 1141-75.

A la vista de los resultados obtenidos en este y en otros trabajos, teniendo en cuenta las condiciones de conservación de los recipientes con las probetas (recinto no climatizado) y con el fin de ampliar el campo a otras disoluciones agresivas, se ha determinado la evolución de los coeficientes de corrosión Koch-Steinegger cuando las diversas series de probetas de mortero $(1: 3)$ se sumergen durante $56-90-180-360-540-720-900$ y 1.080 dias, después del periodo de curado $(1+21$ días $)$, en las siguientes disoluciones:

Disolución 1. Disolución saturada de yeso.

Disolución 3. Disolución de $\left.\mathrm{MgSO}_{4} \cdot 7 \mathrm{H}_{2} \mathrm{O}\right)$, p.a. $(3,22 \mathrm{~g} / l)$.

Disolución 4. 'Disolución de $\mathrm{MgSO}_{4} \cdot 7 \mathrm{H}_{2} \mathrm{O}$, p.a. $(32,2 \mathrm{~g} / l)$.

Disolución 5. Disolución saturada de yeso $+\mathrm{MgSO}_{4} \cdot 7 \mathrm{H}_{2} \mathrm{O}$, p.a. $(3,22 \mathrm{~g} / l)$.

Disolución 6. Disolución saturada de yeso $+\mathrm{MgSO}_{4} \cdot 7 \mathrm{H}_{2} \mathrm{O}$, p.a. $(32,2 \mathrm{~g} / l)$.

Disolución 8. Agua de mar artificial ASTM D 1141-75 y, además,

Disolución 0. Agua potable filtrada.

Los recipientes con las probetas se han conservado en un laboratorio a $20 \pm 2^{\circ} \mathrm{C}$. 


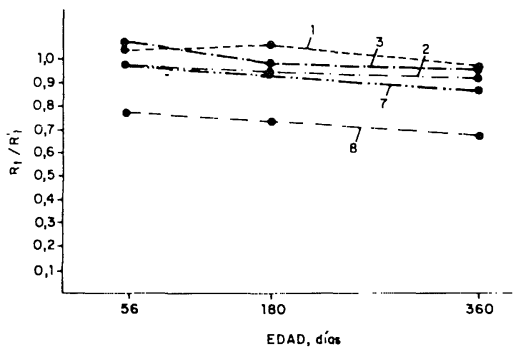

Fig. 4.-Evolución de los coeficiente de corrosión Koch-Steinegger.

1. Disolución saturada de sulfato de calcio.

2. Disolución de $\mathrm{Na}_{2} \mathrm{SO}_{4}(2,1 \mathrm{~g} / l)$.

3. Disolución de $\left.\mathrm{MgSO}_{4} .7 \mathrm{H}_{2} \mathrm{O}\right)(3,22 \mathrm{~g} / l)$.

7. Disolución de $\mathrm{Na}_{2} \mathrm{SO}_{4}(3,5 \mathrm{~g} / l)+\mathrm{NaCl}(20,0 \mathrm{~g} / l)$.

8. Agua de mar artificial ASTM D 1141-75.

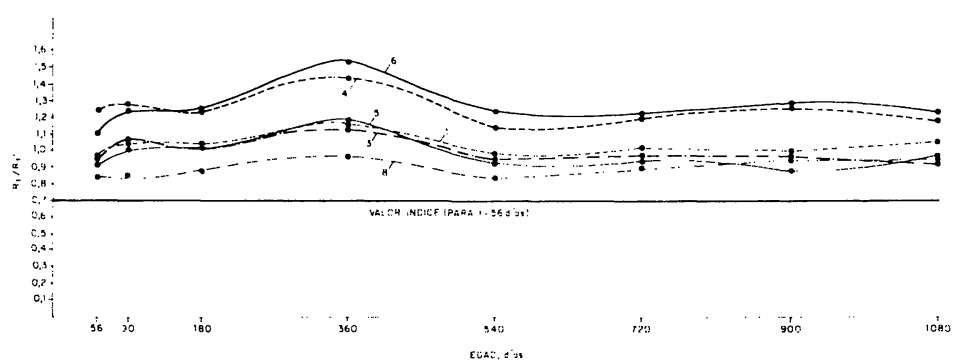

Fig. 5.-Evolución de los coeficientes de corrosión Koch-Steinegger.

1. Disolución saturada de sulfato de calcio.

3. Disolución de $\left.\mathrm{MgSO}_{4} .7 \mathrm{H}_{2} \mathrm{O}\right)(3,22 \mathrm{~g} / \mathrm{l})$.

4. Disolución de $\mathrm{MgSO}_{4} .7 \mathrm{H}_{2} \mathrm{O}(32,2 \mathrm{~g} / l)$.

5. Disolución saturada de sulfato de calcio $+\mathrm{MgSO}_{4} \cdot 7 \mathrm{H}_{2} \mathrm{O}(3,22 \mathrm{~g} / l)$.

6. Disolución saturada de sulfato de calcio $+\mathrm{MgSO}_{4} .7 \mathrm{H}_{2} \mathrm{O}(32,2 \mathrm{~g} / l)$.

8. Agua de mar artificial ASTM D 1141-75.

En la figura 5 se han representado los valores obtenidos (coeficientes de corrosión) en función del tiempo de conservación. En dicha figura se puede observar que:

a) Todos los valores - a las distintas edades - son superiores al valor especificado para calificar a un cemento como resistente frente a un determinado medio agresivo $\left(R_{\mathrm{t}} / R_{\mathrm{t}}^{\prime}=0,70\right.$, para $t=22+56$ dias).

b) Dichos valores son superiores a 1,00 en los casos de las probetas sumergidas en la disolución saturada de yeso más $32,2 \mathrm{~g} / l$ de $\mathrm{MgSO}_{4} \cdot 7 \mathrm{H}_{2} \mathrm{O}$ y en la disolución de $\mathrm{MgSO}_{4} \cdot 7 \mathrm{H}_{2} \mathrm{O}$ $(32,2 \mathrm{~g} / l)$; aproximadamente, igual a 1,00 , con pequeñas variaciones, en los casos de las probetas sumergidas en la disolución saturada de yeso, en la disolución de $\mathrm{MgSO}_{4} \cdot 7 \mathrm{H}_{2} \mathrm{O}$ $(3,22 \mathrm{~g} / l)$ y en la disolución saturada de yeso más $3,22 \mathrm{~g} / l$ de $\mathrm{MgSO}_{4} .7 \mathrm{H}_{2} \mathrm{O}$ y menores de 1,00 en el caso de las probetas sumergidas en agua de mar artificial ASTM D 1141-75.

c) Los valores mayores corresponden a las series de probetas sumergidas en la disolución saturada de yeso que tiene $32,2 \mathrm{~g} / l$ de $\mathrm{MgSO}_{4} \cdot 7 \mathrm{H}_{2} \mathrm{O}$, junto con la de las series de probetas sumergidas en la disolución de $\mathrm{MgSO}_{4} .7 \mathrm{H}_{2} \mathrm{O}(32,2 \mathrm{~g} / l)$ y los menores a las de las probetas sumergidas en agua de mar artificial ASTM D 1141-75.

De acuerdo con los valores obtenidos, en este método, se puede establecer el siguiente orden de resistencia química del cemento frente a las disoluciones agresivas estudiadas:

Disolución saturada de yeso más $32,2 \mathrm{~g} / l$ de $\mathrm{MgSO}_{4} \cdot 7 \mathrm{H}_{2} \mathrm{O}>$ Disolución de $\mathrm{MgSO}_{4} \cdot 7 \mathrm{H}_{2} \mathrm{O}$ $(32,2 \mathrm{~g} / l)>$ Disolución saturada de yeso $\simeq \mathrm{y}>$ Disolución de $\mathrm{MgSO}_{4} \cdot 7 \mathrm{H}_{2} \mathrm{O}(3,22 \mathrm{~g} / l) \simeq \mathrm{Di}$ solución saturada de yeso más $3,22 \mathrm{~g} / l$ de $\mathrm{MgSO}_{4} .7 \mathrm{H}_{2} \mathrm{O}>$ Agua de mar artificial ASTM D 1141-75.

Dichos coeficientes de corrosión son consecuencia de los valores de las resistencias mecánicas a flexotracción de las diversas series de probetas. Dichos valores de las resistencias de las series de probetas sumergidas en las disoluciones agresivas, con relación a los valores correspondientes a las series conservadas bajo agua potable filtrada, son:

a) mayores, a todas las edades, para el caso de las probetas sumergidas en la disolución saturada de yeso $+32,2 \mathrm{~g} / \mathrm{l}$ de $\mathrm{MgSO}_{4} .7 \mathrm{H}_{2} \mathrm{O}$ y en la disolución de $\mathrm{MgSO}_{4} .7 \mathrm{H}_{2} \mathrm{O}$ $(32,2 \mathrm{~g} / \mathrm{l})$. Los valores de dichas resistencias de las probetas sumergidas en la disolución saturada de yeso son, también, mayores, excepto para dos edades que son aproximadamente iguales. 
b) del mismo orden, o con variaciones en más o en menos para determinadas edades, en los casos de las series de probetas sumergidas en la disolución de $\mathrm{MgSO}_{4} \cdot 7 \mathrm{H}_{2} \mathrm{O}(3,22 \mathrm{~g} / l)$ y de la disolución saturada de yeso $+3,22 \mathrm{~g} / l \mathrm{deMgSO}_{4} .7 \mathrm{H}_{2} \mathrm{O}$,

c) menores, con tendencia a conseguir valores del mismo orden, para el caso de las series de probetas conservadas bajo agua de mar artificial ASTM D 1141-75.

\section{CONCLUSIONES}

\section{Primera}

El cemento estudiado, objeto de este trabajo, resiste la acción del sulfato de calcio (método de Le Chatelier-Anstett y ASTM C 452-73) presentando, a lo largo del período de tiempo considerado (hasta 1 año), valores del mismo orden que los obtenidos a las primeras edades (28 días para el primer método y 14 días para el segundo).

\section{Segunda}

Los valores de los coeficientes de corrosión Koch-Steinegger de todas las series de probetas sumergidas en las diversas disoluciones agresivas, estudiadas en este trabajo, son superiores a 0,70 . Los valores más altos (superiores a la unidad) corresponden a las series de probetas sometidas a la acción de las disoluciones saturadas de sulfato de calcio $+32,2 \mathrm{~g} / l \mathrm{de} \mathrm{MgSO}_{4} .7 \mathrm{H}_{2} \mathrm{O}$ y de $\mathrm{MgSO}_{4} .7 \mathrm{H}_{2} \mathrm{O}(32,2 \mathrm{~g} / l)$ y los valores más bajos (comprendidos entre 0,81 y 0,97$)$ a las series de probetas conservadas en agua de mar artificial ASTM C 452-75.

\section{Tercera}

Las variaciones de longitud experimentadas por las series de probetas sumergidas en las disoluciones agresivas, excepto para el agua de mar artificial, son del mismo orden que las de las series conservadas en agua potable filtrada (método de Wittekindt); en el caso de las probetas sometidas a la acción del agua de mar experimentan una retracción comprendida entre 0,006 y $0,19 \mathrm{~mm} / \mathrm{m}$, en los diversos periodos de tiempo estudiados.

\section{Cuarta}

La composición de las disoluciones agresivas estudiadas influye en los valores de los coeficientes de corrosión, lo que ha permitido establecer una graduación del comportamiento del cemento mencionado frente a dichas disoluciones.

\section{BIBLIOGRAFIA}

(1) GASPAR-TEBAR, D. y SAGRERA-MORENO, J. L. (1980): Resistencia quimica del hormigón: V. Acción del agua de mar: influencia de la adición de escoria a un cemento portland resistente al yeso. Evolución de las resistencias mecánicas a flexotracción y del coeficiente de corrosión. Materiales de Construcción, julio-agosto-septiembre, 1979, 59-79.

(2) SAgRERA-MORENO, J. L. y GASPAR-TEBAR, D. (1982): Resistencia quimica del hormigón: XVII. Acción de una disolución saturada de yeso: influencia de la adición de escoria a un cemento portland resistente al yeso. Evolución de las resistencias mecánicas a flexotracción y de los coeficientes de corrosión. Materiales de Construcción, julio-agosto-septiembre, 187, 41-54.

(3) SAGRERA-MORENO, J. L. y GASPAR-TEBAR, D. (1981): Resistencia quimica del hormigón: X. Influencia de la adición de escoria a un cemento portland resistente al yeso. Estudio por DRX del sistema cemento 2/escoria-agua potable filtrada. Materiales de Construcción, abril-mayo-junio, 182, 11-22. 
(4) GASPAR-TEBAR, D. y SAGRERA-MORENO, J. L. (1982): Resistencia quimica del hormigón: XVIII. Acción del agua de mar: influencia de la adición de escoria a un cemento portland resistente al yeso. Estudio por DRX. Materiales de Construcción, octubre-noviembre-diciembre, 188, 37-58.

(5) SAGRERA-MORENO, J. L. y GASPAR-TEBAR, D. (1984): Resistencia quimica del hormigón: XXIII. Influencia de la adición de escoria a un cemento portland resistente al yeso. Estudio de la concentración iónica del sistema cemento 2/escoria-agua potable filtrada. Materiales de Construcción, abril-mayo-junio, 194, 43-51.

(6) GASPAR-TEBAR, D. y SAGRERA-MORENO, J. L. (1984): Resistencia química del hormigón: XXIV. Influencia de la adición de escoria a un cemento portland resistente al yeso. Estudio de la concentración iónica del sistema cemento 2/escoria-agua de mar artificial. Materiales de Construcción, octubre-noviembre-diciembre, 196, 49-66.

(7) TAKASHIMA, S. and AMANO, F. (1960): Some studies on lower calcium silicates in portland cement. Rev. of the fourteenth General Meeting; Tokyo, págs. 19-22.

(8) ASTM D 1141-75: Standard Specification for Substitute Ocean Water; 1978 Annual Book of ASTM Standards, Part 31, págs. 909-911.

(9) SAGRERA-MORENO, J. L. y GASPAR-TEBAR, D. (1980): Resistencia quimica del hormigón: IV. Acción del agua de mar: influencia de la adición de escoria a un cemento portland de alta resistencia inicial. Evolución de las resistencias mecánicas a flexotracción y del coeficiente de corrosión. Materiales de Construcción, abril-mayo-junio, $178,20-21$.

(10) Pliego de Prescripciones Técnicas Generales para la Recepción de Cementos (RC-75); BOE n.o 206 y n. 207 de 28-agosto-1975 y 29-agosto-1975, respectivamente.

(11) GASPAR-TEBAR, D. y SAGRERA-MORENO, J. L. (1979): Resistencia quimica del hormigón: III. Acción del agua de mar. Influencia de la adición de escoria al cemento portland $\left(1 .^{a}\right.$ parte $)$. Materiales de Construcción, abrilmayo-junio, 174, 48.

(12) BLONDIAU, L. (1961): Considérations diverses relatives á l'essai de résistance chimique au sulfate de calcium suivant le processus Le Chatelier-Anstett. Rev. des Matériaux de Construction, mars, 546, 189-200.

(13) Norma ASTM C 452-75: Standard Method of Test for Potential Expansion of Portland Cement Mortars Exposed to Sulfate.

(14) GASPAR-TEBAR, D. y SAGRERA-MORENO, J. L. (1977): Resistencia quimica del hormigón: I. Estudio de dos cementos portland frente al agua de mar. Materiales de Construcción, 168, 33-60.

(15) GARCIA DE PAREDES, P. (1967): Inalterabilidad de los conglomerantes frente al ataque de los sulfatos. Cuadernos de Investigación del IETcc, diciembre, 36 págs.

\section{RECONOCIMIENTO}

Nuestro más sincero agradecimiento a las personas del Equipo de Durabilidad del IETcc: Amalia Rodriguez Pereira, Lucila López Solana, Felipe Cantero Palacios y Manuel Cantero Palacios por su valiosa colaboración en la realización de este trabajo. 